\title{
Alteração na atenção e o tratamento da dor do câncer*
}

\author{
ALTERATION IN ATTENTION AND CANCER PAIN TREATMENT
}

ALTERACIÓN EN LA ATENCIÓN Y EL TRATAMIENTO DEL DOLOR DE CÁNCER

\author{
Geana Paula Kurita ${ }^{1}$, Cibele Andrucioli de Mattos Pimenta ${ }^{2}$, \\ José Oswaldo de Oliveira Júnior ${ }^{3}$, Ricardo Caponeiro ${ }^{4}$
}

\begin{abstract}
RESUMO
Os danos à vida diária que a alteração cognitiva pode provocar motivaram a elaboração deste estudo, cujo objetivo foi analisar o impacto do tratamento da dor com opióides sobre a atenção. Os doentes foram divididos em grupos que recebiam $(n=14)$ e não recebiam opióides $(n=12)$. Foram feitas três entrevistas, utilizando-se o Trail Making Test e o Digit Span Test, que avaliam a atenção. Os grupos foram homogêneos nas variáveis sociodemográficas, dor e depressão; não foram homogêneos no índice de Karnofsky e no recebimento de analgésicos adjuvantes. Os doentes sem opióides tiveram melhor desempenho no Digit Span Test-ordem inversa, na segunda avaliação $(p=0,29)$ e não foram observadas diferenças no Trail Making Test. As alterações observadas foram limitadas, mas, enquanto novos estudos não confirmem os achados, doentes, profissionais e cuidadores devem ser alertados dos possíveis efeitos deletérios dos opióides sobre a função cognitiva.
\end{abstract}

\section{DESCRITORES}

Dor.

Neoplasias.

Analgésicos opióides.

Atenção.

Transtornos cognitivos.

\begin{abstract}
Cognitive impairment can negatively impact daily routine. This fact encouraged the elaboration of this study, which analyzed the impact of pain treatment with opioids on attention. Patients were divided into groups that were given $(n=14)$ and were not given opioids $(n=12)$. Three interviews were conducted to assess attention using the Trail Making Test and the Digit Span Test. The groups were homogeneous regarding socio-demographic variables, pain and depression; they were not homogeneous regarding the Karnofsky scale and the use of adjuvant analgesics. The patients in treatment without opioids had better performance in the Digit Span Test - reverse order, in the second assessment ( $p=0.29$ ); no differences were observed on the Trail Making Test. The observed impairments were limited, but while new studies do not confirm the findings, patients, professionals and caregivers must be alert about the possible deleterious effects of opioids on the cognitive function.
\end{abstract}

KEY WORDS
Pain.
Neoplasms.
Analgesics, opioid.
Attention.
Cognition disorders.

\section{RESUMEN}

Los daños a la vida diaria que la alteración cognoscitiva puede causar motivaron la elaboración de este estudio, cuyo objetivo fue analizar el impacto del tratamiento del dolor con los opioides en la atención. Los enfermos fueron divididos en grupos que recibieron $(n=14)$ y no recibieron opioides $(n=12)$. Fueron realizadas tres entrevistas utilizándose el Trail Making Test y el Digit Span Test, que evalúan la atención. Los grupos fueron homogéneos en las variables sociodemográficas, dolor y depresión; no fueron homogéneos en el índice de Karnofsky y en el suministro de analgésicos adyuvantes. Los pacientes sin opioides tuvieron mejor desempeño en el Digit Span Test - reverse order, en la segunda entrevista $(p=0,29)$ y no se observaron diferencias en el Trail Making Test. Las alteraciones observadas fueron limitadas, sin embargo mientras nuevos estudios no confirmen los resultados, pacientes, profesionales y cuidadores deben ser alertados sobre los posibles efectos deletéreos de los opioides en la función cognoscitiva.

\section{DESCRIPTORES}

Dolor.

Neoplasias.

Analgesicos opioides.

Atención.

Trastornos del conocimiento.

*Extraído da tese "Alteração cognitiva e o tratamento da dor oncológica", Escola de Enfermagem, Universidade de São Paulo, 2006. ${ }^{1}$ Enfermeira. Doutora em Enfermagem pela Escola de Enfermagem, Universidade de São Paulo (EEUSP). São Paulo, SP, Brasil. geanakurita@yahoo.com.br 2 Enfermeira. Professora Titular do Departamento de Enfermagem Médico-Cirúrgica da Escola de Enfermagem, Universidade de São Paulo (EEUSP). São Paulo, SP, Brasil. parpca@usp.br ${ }^{3}$ Médico Neurocirurgião. Diretor da Central de Dor e Extereotaxia do Hospital do Câncer da Fundação Antônio Prudente. São Paulo, SP, Brasil. ${ }^{4}$ Médico Oncologista Clínico. Oncologista do Hospital Brigadeiro. São Paulo, SP, Brasil 


\section{INTRODUÇÃO E OBJETIVO}

O adequado controle da dor é tema atual e considerado indicador de qualidade de vida e de assistência. Analgésicos opióides são a pedra angular para o controle da dor do câncer, da dor aguda de moderada a intensa e, algumas vezes, são utilizados em dores crônicas não relacionadas ao câncer. $O$ uso desses fármacos é recomendado pela Organização Mundial de Saúde para o alívio da dor do câncer há décadas, e os benefícios relacionados ao controle da dor são amplamente conhecidos. No entanto, os efeitos dos opióides sobre a função cognitiva é assunto ainda não completamente esclarecido; a literatura sobre o tema é escassa e aponta a necessidade de estudos que analisem o impacto do uso crônico desses analgésicos sobre as funções mentais.

Alterações cognitivas são freqüentes na população de doentes oncológicos, por razões relacionadas à doença e ao tratamento. Há descrições na literatura de que os opióides utilizados no tratamento da dor possam ser responsáveis por tais alterações ${ }^{(1-3)}$. Por outro lado, a dor, por si só, pode causar alterações cognitivas ${ }^{(4)}$. Diante dessa complexa rede de fatores determinantes de alteração cognitiva busca-se aliviar a dor sem adicionar prejuízos à cognição.

Para alguns autores o uso de opióides por doentes com dor crônica parece ser seguro do ponto de vista $\operatorname{cognitivo}{ }^{5}$ e não impossibilitar, por exemplo, a condução de veículos motorizados $^{(6)}$. No entanto, há apreensão sobre a generalização da informação de que os opióides não interferem na função cognitiva de doentes com dor crônica ${ }^{(7)}$.

Altas doses e tratamento prolongado com opióides, desidratação, falência renal, uso de outras drogas psicoativas e idade avançada representam fatores de risco para a indução de neurotoxicidade por opióides (disfunção cognitiva, sedação intensa, alucinação, ataques de mioclonia e hiperalgesia) ${ }^{(8)}$. Doentes tratados pela primeira vez com analgésicos opióides para dor oncológica podem desenvolver sedação transitória ou falência cognitiva. Podem sofrer alucinações visuais ou táteis e mudanças no humor ${ }^{(8)}$. Além disso, alterações na atenção devido ao uso de opióides em doentes com dor crônica foram relatadas por autores ${ }^{(9)} \mathrm{e}$ tal fato é preocupante, pois muitas atividades rotineiras exigem atenção que, se prejudicada, pode ocasionar danos à vida cotidiana. Atenção é um mecanismo cognitivo que permite processar informações, pensamentos ou ações. É a focalização, a concentração da consciência em um objeto ou pensamento ${ }^{(10)}$.

A falta de consenso sobre os efeitos dos opióides na cognição e o aumento na indicação de tais medicamentos para o controle da dor crônica de diferentes etiologias resultaram na elaboração dessa investigação, que teve o objetivo de comparar a atenção/concentração em doen- tes com câncer em tratamento para dor, utilizando ou não opióides.

\section{MÉTODO}

O estudo foi desenvolvido na Central da Dor do Hospital do Câncer A. C. Camargo e no Ambulatório de Oncologia do Hospital Brigadeiro, localizados na cidade de São Paulo. O período de coleta de dados foi entre os meses de fevereiro a outubro de 2005, após a aprovação pelos Comitês de Ética em Pesquisa das referidas instituições.

Foram critérios de inclusão ter idade igual ou superior a dezoito anos, ter no mínimo seis anos de estudo, possuir capacidade de compreensão e de verbalização adequadas no início do tratamento, ter função renal e hepática preservadas, não apresentar sinais clínicos de desidratação, não apresentar dosagem de hemoglobina inferior a $10 \mathrm{~g} / \mathrm{dl}$, não ter história prévia de doença mental, não fazer uso de álcool, não ser usuário de drogas, residir na cidade de São Paulo ou na Grande São Paulo e ter conhecimento de que sua dor era relacionada ao câncer.

A amostra, de conveniência, foi composta por 14 doentes com dor oncológica em tratamento com opióides (Grupo Recebendo Opióide - GRO) e 12 doentes também com dor oncológica, mas em tratamento analgésico sem opióides (Grupo Sem Opióide - GSO). O tipo de opióide diferiu e, para permitir comparações, as doses foram convertidas em dose equivalente de morfina via oral ${ }^{(11)}$.

A proposição era de que os doentes fossem avaliados três vezes ao longo de um mês, com intervalos de uma a duas semanas entre as avaliações, o que ocorreu em parcela dos entrevistados, pois alguns doentes abandonaram o estudo por piora do estado geral. 0 número de doentes que participou em cada avaliação pode ser observado na Tabela 1. As entrevistas foram feitas em ambiente privativo e tranqüilo e os doentes assinaram Termo de Consentimento Livre e Esclarecido.

Os dados sociodemográficos (sexo, estado civil, idade, escolaridade e renda), sobre a dor (duração e intensidade), sobre o uso de medicamentos (quais, doses e freqüências) e sobre hábitos e história pregressa (não consumo de álcool, drogas e ausência de diagnóstico de doença mental) foram obtidos com os doentes. O tipo de tumor, presença e local das metástases, os valores de hemoglobina, uréia, creatinina e enzimas hepáticas foram obtidos do prontuário médico. O Índice de Karnofsky foi avaliado pelas pesquisadoras ${ }^{(12)}$.

Os instrumentos utilizados para avaliação da atenção foram o Digit Span Test e o Trail Making Test ${ }^{(13-14)}$; para a avaliação da depressão foi o Inventário de Depressão de Beck $^{(15)}$ e para avaliar a intensidade da dor utilizou-se a escala numérica de 0 a $10^{(16)}$. 
O Trail Making Test avalia, além da atenção, flexibilidade mental, velocidade de processamento visual e função motora ${ }^{(13-14)}$. Consiste de duas etapas. Na primeira etapa, Parte A, a pessoa deve desenhar com um lápis ou caneta uma linha conectando a série de números dispostos em círculos ao acaso em seqüência numérica, fazendo uma verificação visual simples. Na segunda, Parte $B$, adiciona-se a dimensão de flexibilidade cognitiva, pois a pessoa deve ligar números e letras dentro de círculos em seqüências alternadas, em ordem crescente. O critério de correção é o tempo (em segundos) e quanto maior o tempo utilizado para concluir cada parte, pior o desempenho.

O Digit Span Test avalia a atenção e a memória operacional $^{(13-14)}$. Na aplicação do teste são recitados números para os doentes (que variam de dois a sete dígitos), que os devem repetir em ordem direta e inversa. Se o entrevistado errar dois dígitos do mesmo número o teste deve ser interrompido e os acertos anotados. Escores mais altos indicam melhor desempenho cognitivo.

O Inventário de Depressão de Beck é um instrumento com 21 itens que avalia a presença e a intensidade de humor deprimido, pessimismo, sensação de fracasso, perda de satisfação, sentimentos de culpa, sensação de punição, auto-rancor, auto-acusação, ideação suicida, choro, irritabilidade, isolamento social, indecisão, per- cepção de imagem corporal alterada, inibição para o trabalho, anormalidades do sono, fatigamento, perda de apetite, perda de peso, preocupações somáticas e perda de libido. A pontuação pode variar entre 0 a 63 e escore mais alto indica maior depressão(15).

O Índice de Karnofsky avalia a capacidade funcional por meio 11 itens, que variam entre $0 \%$ (falecido) a 100\% (funcionalidade total) ${ }^{(12)}$.

Para testar diferenças entre os grupos quanto ao sexo, estado civil, local do tumor e presença de metástase foi utilizado o teste Qui-quadrado. O teste de Mann-Whitney foi utilizado para analisar a existência de diferenças no desempenho cognitivo e comparar os grupos quanto as variáveis idade, escolaridade, renda, índice de Karnofski e tempo de dor. Para todos os testes o nível de significância foi determinado em $5 \%$.

\section{RESULTADOS}

Os grupos foram homogêneos quanto ao estado civil, idade, escolaridade, renda familiar, renda per capita, estado de humor e localização do tumor e diferiram quanto ao Índice de Karnofsky e presença de metástases. No GRO a presença de metástase foi maior e o índice de Karnofsky foi pior (Tabela 2). A intensidade da dor foi igual nos dois grupos e variou entre leve e moderada (Tabela 1 ).

Tabela 1 - Distribuição do número de doentes por grupo e da intensidade da dor, em cada uma das três avaliações São Paulo - 2005

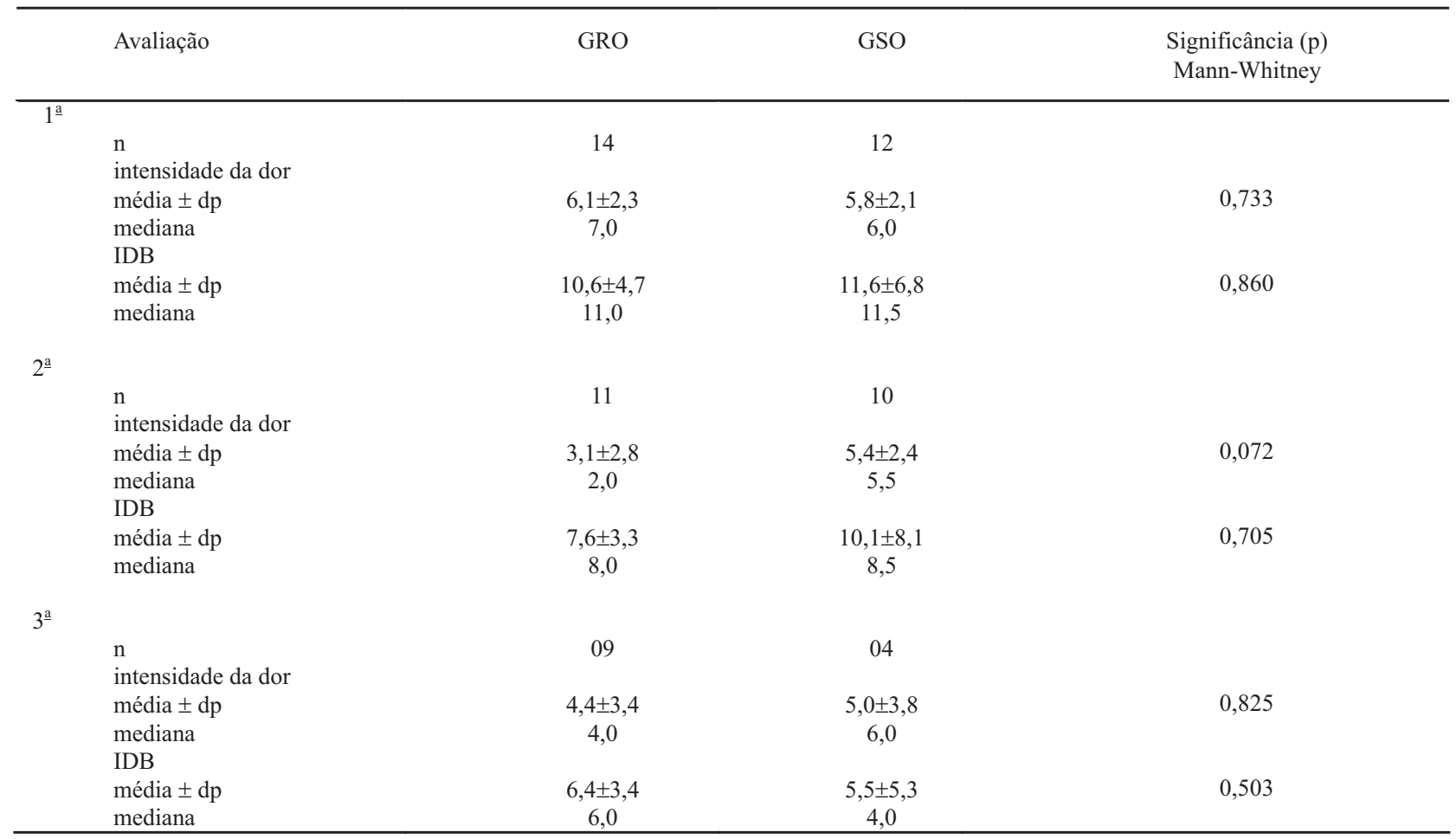

GRO = Grupo Recebendo Opióide GSO = Grupo Sem Opióide dp = desvio padrão IDB = Inventário de Depressão de Beck (escore) 
Tabela 2 - Características sócio-demográficas, da doença e da dor do Grupo Recebendo Opióide (GRO) e do Grupo Sem Opióide (GSO) - São Paulo - 2005

\begin{tabular}{|c|c|c|c|}
\hline Variáveis & GRO $(n=14)$ & GSO $(n=12)$ & $\begin{array}{c}\text { Significância } \\
\text { (p) }\end{array}$ \\
\hline \multicolumn{4}{|l|}{ Sociodemográficas } \\
\hline Sexo & & & Qui-quadrado \\
\hline masculino $\mathrm{n}(\%)$ & $07(50,0 \%)$ & $07(58,4 \%)$ & 0,671 \\
\hline feminino $\mathrm{n}(\%)$ & $07(50,0 \%)$ & $05(41,6 \%)$ & \\
\hline \multicolumn{4}{|l|}{ Estado Civil } \\
\hline com parceiro (a) vivendo no mesmo lar $\mathrm{n}(\%)$ & $10(71,4 \%)$ & $09(75,0 \%)$ & Qui-quadrado \\
\hline sem parceiro (a) vivendo no mesmo lar $\mathrm{n}(\%)$ & $04(28,6 \%)$ & $03(25,0 \%)$ & 0,838 \\
\hline Idade em anos & & & Mann-Whitney \\
\hline média \pm dp & $52,4 \pm 12,9$ & $54,3 \pm 16,9$ & 0,560 \\
\hline mediana & 53,5 & 56,5 & \\
\hline $1^{\circ}$ e $3^{\circ}$ quartil & $34-74$ & $19-76$ & \\
\hline Escolaridade em anos & & & Mann-Whitney \\
\hline média $\pm \mathrm{dp}$ & $11,0 \pm 3,3$ & $10,1 \pm 4,1$ & 0,403 \\
\hline mediana & 11,0 & 8,8 & \\
\hline $1^{\circ}$ e $3^{\circ}$ quartil & $6-16$ & $6-19$ & \\
\hline Renda familiar em salários mínimos & & & Mann-Whitney \\
\hline média $\pm \mathrm{dp}$ & $8,1 \pm 12,4$ & $7,7 \pm 7,5$ & 0,742 \\
\hline mediana & 5,0 & 5,0 & \\
\hline $1^{\circ}$ e $3^{\circ}$ quartil & $1-50$ & $1,3-25$ & \\
\hline Renda per capita em salários mínimos & & & Mann-Whitney \\
\hline média $\pm \mathrm{dp}$ & $2,8 \pm 3,2$ & $2,8 \pm 3,5$ & 0,940 \\
\hline mediana & 1,6 & 1,4 & \\
\hline $1^{\circ}$ e $3^{\circ}$ quartil & $0,5-12,5$ & $0,4-12,5$ & \\
\hline \multicolumn{4}{|l|}{ Doença/dor } \\
\hline Local do tumor & & & Qui-quadrado \\
\hline cabeça/pescoço n $(\%)$ & $05(35,7 \%)$ & $04(33,3 \%)$ & 0,630 \\
\hline tronco n $(\%)$ & $05(35,7 \%)$ & $02(16,7 \%)$ & \\
\hline abdome n (\%) & $03(21,4 \%)$ & $04(33,3 \%)$ & \\
\hline membros n (\%) & $01(7,1 \%)$ & $02(16,7 \%)$ & \\
\hline \multicolumn{4}{|l|}{ Metástase } \\
\hline $\operatorname{sim} n(\%)$ & $07(50,0 \%)$ & $01 \quad(8,3 \%)$ & Qui-quadrado \\
\hline não n (\%) & $07(50,0 \%)$ & $11(91,7 \%)$ & $0,022 *$ \\
\hline \multicolumn{4}{|l|}{ Índice de Karnofski (0 \% a 100\%) } \\
\hline média $\pm d p$ & $66,4 \pm 11,5$ & $82,5 \pm 9,7$ & Mann-Whitney \\
\hline mediana & 65,0 & 85,0 & $0,002 *$ \\
\hline $1^{\circ}$ e $3^{\circ}$ quartil & $50,0-90,0$ & $60,0-90,0$ & \\
\hline \multicolumn{4}{|l|}{ Tempo de dor em meses } \\
\hline média $\pm \mathrm{dp}$ & $15,3 \pm 25,4$ & $8,4 \pm 8,6$ & Mann-Whitney \\
\hline mediana & 6,5 & 4,5 & 0,552 \\
\hline $1^{\circ}$ e $3^{\circ}$ quartil & $1,0-96,0$ & $0,2-24,0$ & \\
\hline
\end{tabular}

GRO = Grupo Recebendo Opióide GSO = Grupo Sem Opióide $d p=$ desvio padrão * $p<0,05$ - estatisticamente significativo.

A dose média de opióides do GRO variou de $69,9 \mathrm{mg}$ $\pm 53,1 \mathrm{mg}$ a $112,8 \mathrm{mg} \pm 62,3 \mathrm{mg}$ por dia. Em ambos os grupos foi relatado o uso de analgésicos adjuvantes (analgésicos simples, neurolépticos, antidepressivos e anticonvul- sivantes), mas esses não puderam ser comparados, pois no GSO houve pequeno número de doentes que recebiam tais medicamentos. 
Tabela 3 - Distribuição das doses de opióides e analgésicos adjuvantes utilizados pelos doentes, nas três avaliações - São Paulo - 2005

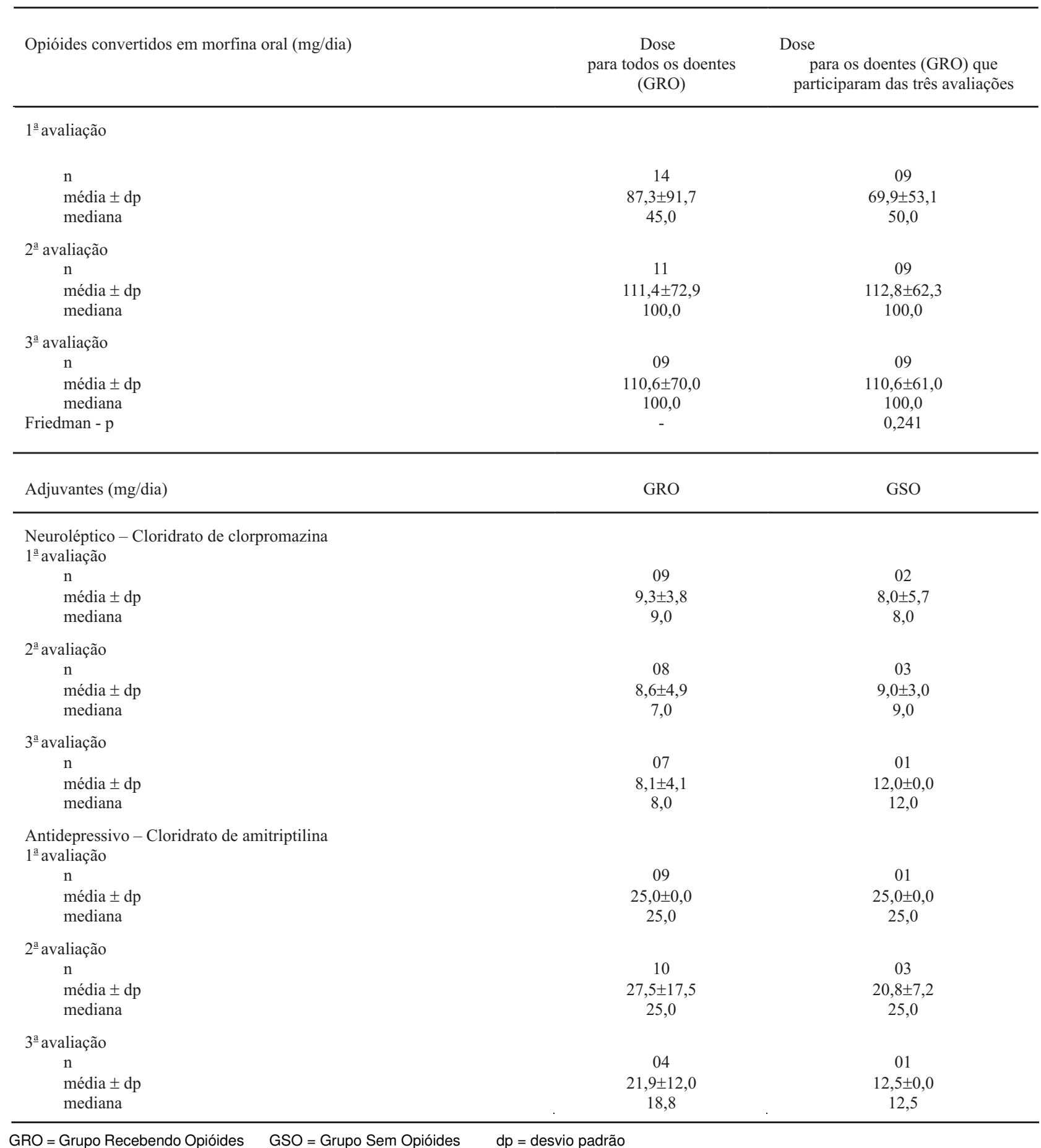

Os grupos diferiram no Digit Span Test - ordem inversa e não diferiram no Trail Making Test (Tabela 4 e 5).

Na análise do Digit Span Test - ordem inversa, observou-se que o GSO teve melhor desempenho que o GRO na segunda avaliação $(p=0,029)$.
A comparação entre os grupos não demonstrou diferenças significativas nos escores do Trail Making Test, em nenhuma das três avaliações. 
Tabela 4 - Comparação dos escores dos doentes obtidos no Digit Span Test, ordem direta e ordem inversa, nas três avaliações - São Paulo - 2005

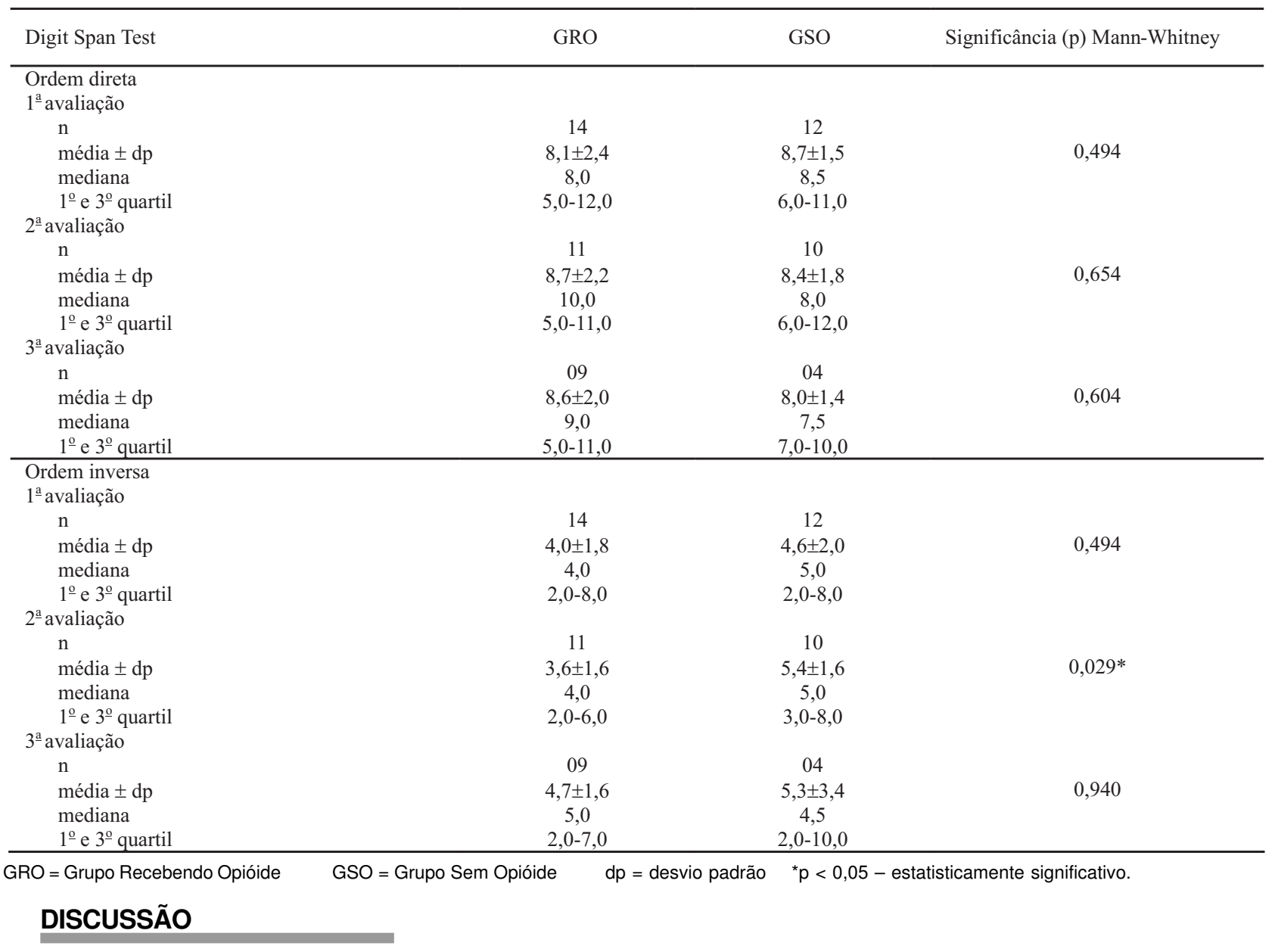

Atenção/concentração é fundamental para a vida diária. Atenção influi na aprendizagem, pois é responsável pela captação, seleção, organização, transformação e fixação temporária e no processamento da informação, que posteriormente será armazenada de maneira permanente. Prejuízos nessas funções podem dificultar o funcionamento da pessoa no dia a dia, comprometer a compreensão e adesão ao tratamento e expor o doente a riscos de acidentes, por exemplo, ao dirigir automóveis ou operar máquinas. $\mathrm{O}$ número de estudos que avaliam a função cognitiva atenção é reduzido ${ }^{(17)}$.

Visando minimizar a lacuna de conhecimento sobre efeitos dos opióides utilizados para o controle da dor do câncer sobre função cognitiva, organizou-se o presente estudo. Observou-se pior desempenho dos doentes que recebiam opióides no Digit Span Test $(p=0,029)$ e não houve diferença quanto ao Trail Making Test (Tabelas 3 e 4).

Da análise dos resultados do Digit Span Test observouse que o prejuízo dos opióides à atenção/concentração foi limitado, pois os doentes que os utilizavam tiveram pior desempenho que aqueles que não os utilizavam somente na segunda avaliação, na parte ordem inversa. Esses da- dos assemelharam-se aos encontrados em estudos que utilizaram o Digit Span Test para avaliar os efeitos dos opióides em doentes com dor crônica de origem não oncológica, nos quais não foram observadas alterações na função atenção/concentração(18-19). Não se encontrou estudo que tenha utilizado o Digit Span Test para avaliar os efeitos dos opióides em doentes com dor crônica neoplásica.

Da análise do Trail Making Test não se observou diferença de desempenho entre os grupos (Tabela 3). Não se encontrou estudo que tenha utilizado o Trail Making Test para avaliar os efeitos dos opióides em doentes com dor crônica neoplásica, mas encontraram-se estudos com doentes com dor crônica não neoplásica ${ }^{(18-22)}$. Em dois estudos houve melhora do tempo de execução do teste nos doentes que utilizavam o opióide ${ }^{(21-2)} \mathrm{e}$ em três não houve diferença ${ }^{(18-20)}$, à semelhança do observado na presente pesquisa. Esses dados indicam que, até o momento, os estudos não relatam prejuízos à função cognitiva decorrentes do uso de opióides ou relatam prejuízos pequenos. Cabe ressaltar que tais dados referem-se ao uso de opióide para o controle da dor crônica e não sobre o uso recreacional ou abusivo desses fármacos. 
Tabela 5 - Comparação dos escores dos doentes obtidos no Trail Making Test, de acordo com o tempo de execução em segundos (s), nas três avaliações - São Paulo - 2005

\begin{tabular}{|c|c|c|c|}
\hline Trail Making Test & GRO & GSO & $\begin{array}{l}\text { Significância (p) } \\
\text { Mann-Whitney }\end{array}$ \\
\hline \multicolumn{4}{|l|}{ Parte A } \\
\hline \multicolumn{4}{|l|}{$1^{\mathrm{a}}$ avaliação } \\
\hline $\mathrm{n}$ & 14 & 12 & \multirow{4}{*}{0,940} \\
\hline média \pm dp (s) & $55,9 \pm 16,1$ & $59,3 \pm 28,1$ & \\
\hline mediana & 56,0 & 60,5 & \\
\hline $1^{\circ}$ e $3^{\circ}$ quartil & $30,0-88,0$ & $30,0-128,0$ & \\
\hline \multicolumn{4}{|l|}{$2^{\mathrm{a}}$ avaliação } \\
\hline $\mathrm{n}$ & 11 & 10 & \multirow{4}{*}{0,605} \\
\hline média \pm dp (s) & $61,7 \pm 25,0$ & $68,6 \pm 54,1$ & \\
\hline mediana & 62,0 & 49,0 & \\
\hline $1^{\circ}$ e $3^{\circ}$ quartil & $31,0-108,0$ & $26,0-201,0$ & \\
\hline \multicolumn{4}{|l|}{$3^{\mathrm{a}}$ avaliação } \\
\hline $\mathrm{n}$ & 09 & 04 & \multirow{4}{*}{0,824} \\
\hline média \pm dp (s) & $56,1 \pm 27,9$ & $48,0 \pm 20,5$ & \\
\hline mediana & 55,0 & 42,5 & \\
\hline $1^{\circ}$ e $3^{\circ}$ quartil & $28,0-119,0$ & $30,0-77,0$ & \\
\hline \multicolumn{4}{|l|}{ Parte B } \\
\hline \multicolumn{4}{|l|}{$1^{\mathrm{a}}$ avaliação } \\
\hline $\mathrm{n}$ & 14 & 12 & \multirow{4}{*}{0,403} \\
\hline média \pm dp (s) & $158,1 \pm 98,3$ & $143,1 \pm 92,1$ & \\
\hline mediana & 128,5 & 103,5 & \\
\hline $1^{\circ}$ e $3^{\circ}$ quartil & $71,0-399,0$ & $63,0-319,0$ & \\
\hline \multicolumn{4}{|l|}{$2^{\mathrm{a}}$ avaliação } \\
\hline $\mathrm{n}$ & $10 *$ & 09 & \multirow{4}{*}{0,133} \\
\hline média \pm dp (s) & $163,2 \pm 123,9$ & $100,7 \pm 59,4$ & \\
\hline mediana & 120,5 & 88,0 & \\
\hline $1^{\circ}$ e $3^{\circ}$ quartil & $60,0-490,0$ & $40,0-211,0$ & \\
\hline \multicolumn{4}{|l|}{$3^{\mathrm{a}}$ avaliação } \\
\hline $\mathrm{n}$ & 09 & 04 & \multirow{4}{*}{0,199} \\
\hline média \pm dp (s) & $177,9 \pm 101,4$ & $100,8 \pm 81,1$ & \\
\hline mediana & 159,0 & 68,0 & \\
\hline $1^{\circ}$ e $3^{\circ}$ quartil & $52,0-408,0$ & $46,0-221,0$ & \\
\hline
\end{tabular}

GRO = Grupo Recebendo Opióide $\quad \mathrm{GSO}=$ Grupo Sem Opióide $\quad \mathrm{dp}=$ desvio padrão *Um doente não conseguiu realizar o teste.

A avaliação cognitiva é área incipiente na enfermagem. A experiência dos enfermeiros em utilizar testes para a avaliação das funções mentais, em geral, restringe-se ao uso de escalas para avaliação do nível de consciência. O Digit Span Test e o Trail Making Test foram escoIhidos por avaliarem habilidades relacionadas à execução de tarefas do dia-a-dia, serem de fácil aplicação e haver grupos nacionais com experiência no uso desses testes, o que permitiu troca de experiências, e por serem tradicionais na área.

O Digit Span Test avalia a atenção, concentração e a memória operacional, isto é a memória necessária para a execução de uma tarefa. Não há publicação nacional sobre a utilização desse teste em doentes com dor crônica, mas há descrição de uso em doentes com distúrbios neurológicos ${ }^{17}$. O Trail Making Test avalia a velocidade de processamento visual e percepção motora, atenção e flexibilidade mental. Não há publicação nacional sobre sua utilização em doentes com dor crônica, mas há estudos que o utilizaram para avaliar outras populações ${ }^{(17)}$.

Outros testes para avaliar a atenção/concentração foram utilizados em doentes com dor crônica em uso de opióides e os resultados foram controversos.
A literatura mundial sobre função cognitiva em doentes com câncer e dor é escassa e o presente estudo é pioneiro no Brasil. A avaliação de alterações cognitivas em doentes com câncer e dor de modo longitudinal, como a realizada no presente estudo, é difícil. O doente poderia estar com múltiplos sistemas acometidos pela doença, o que requereu critérios de inclusão muito restritivos; a evolução da doença, que tende à piora do estado geral e pode requer a introdução de novos tratamentos, aumentar a fadiga e o mal estar e levar os doentes a descontinuar sua participação no estudo, o que ocorreu em parcela dos doentes e prejudicou o tamanho da amostra (Tabela 1). 0 pequeno tamanho da amostra pode ter limitado a expressão das diferenças e é uma limitação deste estudo.

No presente estudo buscou-se controlar variáveis que poderiam influir nas funções cognitivas e para isso os grupos foram comparados em diversos aspectos como depressão, tempo e intensidade da dor, dados sociodemográficos, sobre o tumor e sobre a funcionalidade (índice de Karnofski), entre outros. Os grupos foram homogêneos nas variáveis sexo, estado civil, idade, escolaridade, renda, local do tumor, tempo e intensidade da dor, não foi possível comparálos quanto ao recebimento de antidepressivos e neurolépticos e diferiram no índice de Karnofski (Tabelas 1 e 2). 
Os grupos não puderam ser comparados sobre o recebimento de antidepressivos e neurolépticos, pois o pequeno número de doentes que os recebia não permitiu análise estatística (Tabela 3). Esses fármacos podem influir no desempenho cognitivo, embora não necessariamente de modo negativo, daí o interesse em mantê-los homogêneos nos grupos. Os doentes diferiram quanto ao índice de Karnofski e no número de doentes com metástases, isto é, aqueles do GRO tinham menores índices, o que representa pior funcionalidade (estado geral) e maior número de doentes com metástase, o que também pode contribuir para a piora da funcionalidade. Como o GRO teve pior desempenho no Digit Span Test - ordem inversa, mas também pior Karnofski e maior número de doentes com metástases, é possível que tenha ocorrido sobreposição de fatores (opióides e funcionalidade prejudicada) o que limita a distinção de que efeitos se devem ao opióide ou ao estado geral.

O presente estudo apresenta contribuição original para as áreas de controle da dor e avaliação cognitiva. Frente às limitações do estudo e às pequenas diferenças observadas na função cognitiva entre os grupos, as conclusões

\section{REFERÊNCIAS}

1. Pita G. Disturbances in recent memory and behavioral changes caused by the treatment with intraventricular morphine administration (IVM) in severe cancer pain. Hum Psychopharmacol. 1998;13(6):315-23.

2. Sjøgren $P$, Banning $A$. Pain, sedation and reaction time during long-term treatment of cancer patients with oral and epidural opioids. Pain. 1989;39(1):5-11.

3. Vainio A, Ollila J, Matikainen E, Rosenberg P, Kalso E. Driving ability in cancer patients receiving long-term morphine analgesia. Lancet. 1995;346(8976):667-70.

4. Hart RP, Wade JB, Martelli MF. Cognitive impairment in patients with chronic pain: the significance of stress. Curr Pain Headache Rep. 2003;7(2):116-26.

5. Zacny JP. A review of the effects of opioids on psychomotor and cognitive functioning in humans. Exp Clin Psychopharmacol. 1995;3(4):432-66.

6. Kress HG, Kraft B. Opioid medication and driving ability. Eur J Pain. 2005;9(2):141-4.

7. Chapman SL, Byas-Smith MG, Reed BA. Effects of intermediate and long-term use of opioids on cognition in patients with chronic pain. Clin J Pain. 2002;18(4 Suppl): S83-90.

8. Daeninck PJ, Bruera E. Opiod use in cancer pain: is a more liberal approach enhancing toxicity? Acta Anaesthesiol Scand. 1999;43(9):924-38. devem ser cautelosas. Observou-se piora na atenção/concentração no grupo que recebeu opióides, mas essa diferença foi pequena pois ocorreu somente na segunda avaliação e apenas em parte de um dos testes. Tais achados não permitem afirmar que os opióides utilizados para o controle da dor crônica oncológica acarretaram importantes prejuízos à função mental. Considerando a restrita literatura existente e as graves repercussões que modificações nas funções cognitivas como, por exemplo, modificações na atenção, podem trazer aos doentes, novas investigações devem ser realizadas.

\section{CONCLUSÃO}

As alterações da função cognitiva atenção, em doentes que utilizavam opióides para o controle da dor do câncer foram limitadas, ocorreram apenas no Digit Span Test - ordem inversa, na segunda avaliação. No entanto, frente à carência de estudos na área e aos danos que podem ocasionar, doentes, profissionais e cuidadores devem estar atentos aos possíveis efeitos deletérios dos opióides sobre a função cognitiva.

9. Sjøgren $P$, Thomsen $A B$, Olsen AK. Impaired neuropsychological performance in chronic nonmalignant pain patients receiving long-term oral opioid therapy. J Pain Symptom Manage. 2000;19(2):100-8.

10. Gazzaniga MS, Ivry RB, Mangun GR. Cognitive neuroscience: the biology of the mind. $2^{\text {nd }}$ ed. Nova York: WW Norton; 2002.

11. Twycross RG. Opioids. In: Wall PD, Melzack R, editors. Textbook of pain. $4^{\text {th }}$ ed. Toronto: Churchill Livingstone; 1999. p. 943-62.

12. Schag CC, Heinrich RL, Ganz PA. Karnofsky performance status revisited: reliability, validity, and guidelines. J Clin Oncol. 1984;2(3):187-93.

13.Lezak M, Howieson DB, Loring DW. Neuropsychological assessment. $4^{\text {th }}$ ed. New York: Oxford University Press; 2004.

14. Spreen O, Strauss E. A compendium of neuropsychological tests: administration, norms, and commentary. $2^{\text {nd }}$ ed. New York: Oxford University Press; 1998.

15. Gorenstein L, Andrade L. Inventário de depressão de Beck: propriedades psicométricas da versão em português. Rev Psiq Clin. 1998;25(n.esp):245-50.

16. Paice JA, Cohen FL. Validity of a verbally administered numeric rating scale to measure cancer pain intensity. Cancer Nurs. 1997;20(2):88-93. 
17. Nitrini R, Caramelli $P$, Bottino $C M C$, Damasceno BP, Bruck SMD, Anghinah R. Diagnóstico de doença de Alzheimer no Brasil: avaliação cognitiva e funcional. Recomendações do Departamento Científico de Neurologia Cognitiva e do Envelhecimento da Academia Brasileira de Neurologia. Arq Neuropsiquiatr. 2005;63(3-A):713-9.

18. Haythorthwaite JA, Menefee LA, Quatrano-Piacentini AL, Pappagallo M. Outcome of chronic opiod therapy for non-cancer pain. J Pain Symptom Manage. 1998; 15(3): 185-94.

19. Tassain V, Attal N, Fletcher D, Brasseur L, Dégieux $P$, Chauvin $M$, Bouhassira $D$. Long term effects of oral sustained release morphine on neuropsychological performance in patients with chronic non-cancer pain. Pain. 2003;104(1/2):389-400.
20. Galski T, Williams B, Ehle HT. Effects of opioids on driving ability. J Pain Symptom Manage. 2000;19(3): 200-8.

21. Jamison RN, Schein JR, Vallow S, Ascher S, Vorsanger GJ, Katz NP. Neuropsychological effects of long-term opiod use in chronic pain patients. J Pain Symptom Manage. 2003;26(4):913-21.

22. Menefee LA, Frank ED, Crerand C, Jalali S, Park J, Sanschagrin $K$, et al. The effects of transdermal fentanyl on driving, cognitive performance, and balance in patients with chronic nonmalignant pain conditions. Pain Med. 2004;5(1):42-9. 